\title{
Questes
}

\section{Le cerf qui lève les cornes et le léopard gonflé de sang humain}

(Lyon, Bibliothèque municipale, ms 768)

\section{Julien Abed}

\section{(2) OpenEdition}

\section{Journals}

Édition électronique

URL : http://journals.openedition.org/questes/1654

DOI : $10.4000 /$ questes. 1654

ISSN : 2109-9472

\section{Éditeur}

Les Amis de Questes

Édition imprimée

Date de publication : 15 mars 2008

Pagination : 50-62

ISSN : 2102-7188

\section{Référence électronique}

Julien Abed, «Le cerf qui lève les cornes et le léopard gonflé de sang humain », Questes [En ligne], 14 | 2008, mis en ligne le 01 janvier 2014, consulté le 02 mai 2019. URL : http:// journals.openedition.org/questes/1654; DOI : 10.4000/questes.1654 


\title{
Le cerf qui lève les cornes et le léopard gonflé de sang humain
}

\author{
(Lyon, Bibliothèque municipale, ms 768)
}

\section{Julien ABED}

L'engouement pour les prophéties de Merlin a familiarisé le public lettré du Moyen Âge avec la description de la violence humaine par des métaphores animales. Ce mode d'expression, antérieur au texte de Geoffroy de Monmouth (vers 1135), trouve déjà des racines dans le style biblique ${ }^{1}$. Je voudrais donner une nouvelle illustration de ce phénomène avec une prophétie inédite de la sibylle Tiburtine insérée dans un manuscrit du Pèlerinage de vie humaine de Guillaume de Diguleville ${ }^{2}$. Le décryptage de son symbolisme permet d'y lire des allusions aux événements qui ont opposé France et Angleterre au XIV ${ }^{\mathrm{e}}$ siècle.

De prime abord, le texte ne fait pas attendre un tel débordement de violences animales. Il s'ajoute aux cinq versions françaises en prose déjà connues de la prophétie de la sibylle Tiburtine, et se conforme comme elles au scénario latin de la prophétie, diffusée par plus de 115 manuscrits ${ }^{3}$. Le début du

\footnotetext{
${ }^{1}$ Par exemple Daniel, 7, Apocalypse, 12-13, comme le rappelle Michael CURLEY, « Animal Symbolism in the Prophecies of Merlin ", dans Beasts and Birds of the Middle Ages : The Bestiary and Its Legacy, éd. Willene B. CLARK et Meradith T. MCNunN, Philadelphia, University of Pennsylvania Press, 1989 (coll. The Middle Ages), p. 151-63.

${ }^{2}$ L'existence de ce texte inédit dans le manuscrit Lyon, Bibliothèque municipale 768, m'a été signalée par GÉRALDINE VEYSSEYRE, qui m'a généreusement donné, outre la matière de cette brève présentation, sa transcription. Deux textes prophétiques en fait se suivent, un premier attribué à la sibylle Tiburtine, et un second à Joachin de Flore. Ils sont intercalés aux $\mathrm{f}^{\mathrm{O}} 95^{\mathrm{vb}}$ $99^{\mathrm{v}}$, entre le Pèlerinage de vie humaine (première rédaction) et le Pèlerinage de l'âme (version longue). Le manuscrit est daté du XIV siècle.

${ }^{3}$ Voir l'introduction de La Prophétie de la Sibylle Tiburtine, édition des manuscrits BnF fr. 375 et Rennes BM fr. 593, éd. Jeanne BAROIN et Josiane HAFfen, Paris, Les Belles Lettres, 1987 (coll. Annales littéraires de l'Université de Besançon, 355). Le nombre de manuscrits, longtemps surévalué, est justifié dans l'étude citée plus bas d'Anke Holdenried.
} 
texte renvoie à une source: "Es Sages tiennent en une ystoire que l'en apelle Panthaleon le tesmoigne en son livre qu'il fut jadis $X$ sebiles ... », $\left.\mathrm{f}^{\circ} 95^{\mathrm{v}}\right)$. Le Panthéon de Godefroi de Viterbe contient en effet la Sibylla Tiburtina, en prose latine, insérée au cœur de la chronique (vers 1185) ${ }^{4}$.

Le canevas de la prophétie est respecté dans ses grandes lignes. Il repose sur le récit d'un songe au cours duquel cent sénateurs romains voient défiler neuf soleils de couleurs différentes. Pantois, ils font appel à l'illustre sibylle Tiburtine, qui leur expose au sommet d'une montagne la signification de leur rêve : les neuf soleils représentent neuf générations de l'humanité, depuis l'âge d'or jusqu'à la fin du temps. La prophétesse évoque, vers la quatrième génération (postérieure à ses destinataires fictifs - les sénateurs - mais antérieure évidemment aux lecteurs de la prophétie), la naissance d'un Sauveur.

Dans les versions latines habituelles, mais également dans les traductions françaises connues, la sibylle Tiburtine déroule, après la venue du Christ, une série impressionnante de règnes, en utilisant des initiales pour désigner rois et empereurs ${ }^{5}$. La chronique du temps futur qu'est la prophétie politique permet de crypter ainsi une partie du passé ( $\mathrm{k}$ renvoyant au règne de Charlemagne, L à Louis le Pieux ou à Lothaire, trois o désignant les empereurs Otton, etc), et d'esquisser une partie du futur, par le biais de ces énigmatiques initiales. C'est cette aride partie qui a longtemps fixé l'attention des chercheurs, depuis les médiévistes allemands de la fin du XIX ${ }^{\mathrm{e}}$ siècle, qui avaient soigneusement

\footnotetext{
${ }^{4}$ édition partielle dans Godefroi De Viterbe, Pantheon, éd. Georg WAitz, dans Monumenta Germaniae historica, Scriptorum, XXII, 1872, p. 145-147. Pour l'intégralité de la prophétie, il faut se reporter Fr. GERSS, "Die Sibylle Gottfrieds von Viterbo in anderer Gestalt», Forschungen zur deutschen Geschichte, 19 (1879), p. 375-396.

${ }^{5}$ Pour ce texte, voir Ernst SACKUR, Sibyllinische Texte und Forschungen. Pseudomethodius, Adso und die Tiburtinische Sibylle, Halle, Max Niemeyer, 1898. Les études ont depuis considérablement compliqué notre compréhension de la tradition manuscrite, si bien que les études récentes ne s'engagent plus dans la voie d'une édition. Pour une traduction française à partir de cette édition, voir La Fin des temps, terreurs et prophéties au Moyen Âge, éds. Claude CAROzZI et Huguette TAVIANI-CAROzZI, Paris, Flammarion, 1999 ( ${ }^{\text {ère }}$ éd. Paris, Stock, 1982) - traduction française de la Tiburtina, p. 127-136.
} 
analysé le motif de l'Empereur de la Fin des Temps et confisqué la lecture du texte au seul profit du sens historique de la prophétie. En 2006, Anke Holdenried est revenue de manière décisive sur cette interprétation univoque et a tenté de montrer, par l'analyse des manuscrits (provenance, diffusion, organisation, rubriques, notes marginales), que la Sibylla Tiburtina avait également pu être copiée à des fins morales ou édifiantes ${ }^{6}$. Elle a suggéré que cette succession d'initiales ne faisait peut-être pas sens pour tous les copistes, qui saisissaient en revanche la transparente allusion au Christ.

Il est significatif que le copiste du manuscrit de Lyon ne traduise pas ce passage, et résume avec désinvolture ce long développement en narrativisant les propos sibyllins : «Et aprés Sebile expont le nom des rois et des emperours qui doivent regner en terre jusques a Antechrist, en metant soulement la I letre du nom de chascun: par I, Julius Cesar, par K, Karles, par L, Lois». Cette intervention, qui escamote une partie essentielle de la prophétie, rappelle que la diffusion des textes prophétiques médiévaux en passe souvent par l'omission, la lacune, le rapiéçage, dès lors qu'il s'agit de traduire, c'est-à-dire de comprendre, au sens littéral, le discours divinatoire. Ces phénomènes ont été bien étudiés, à la lumière des prophéties de Merlin notamment ${ }^{7}$.

Néanmoins, dans la brèche ainsi créée par le copiste s'engouffrent de nouvelles prophéties, où interviennent les animaux dont je vais maintenant

\footnotetext{
${ }^{6}$ Anke HoldenrIED, The Sibyl and Her Scribes : Manuscripts and Interpretation of the Latin Sibylla Tiburtina, c. 1050-1500, Burlington, Ashgate, 2006 (Church, Faith and Culture in the Medieval West). Voir aussi son article "Aspects of the English Reception of the Sibylla Tiburtina : Prophecy and Devotion », dans Nigel Morgan (éd.), Prophecy, Apocalypse and the Day of Doom. Harlaxton Medieval Studies 12, Proceedings of the Seventeenth Harlaxton Symposium July 2000, Donington, Shaun Tyas, 2004, p. 118-138.

${ }^{7}$ Par exemple : Richard TRACHSLER, «Des Prophetiae Merlini aux Prophecies Merlin, ou comment traduire les vaticinations de Merlin ", Actes du colloque "Translatio médiévale" organisé par le Laboratoire d'études et travaux sur les translations européennes et le Centre de philologie et de linguistique romane (Mulhouse, 11-12 mai 2000), éd. Claudio GALDERISI et Gilbert SALMON, Paris, Société de langue et de littérature médiévales d'oc et d'oïl, 2000 (Perspectives médiévales, supplément au n 26), p. 105-124.
} 
parler. Notons que ces prédictions n'ont pas de rapport avec les prophéties politiques attribuées à la sibylle Érythréenne ${ }^{8}$, et sont pour l'instant de source inconnue. Elles me semblent librement inspirées des paragraphes 34-44 de la Prophetia Merlini de Geoffroy ${ }^{9}$, sans qu'il me soit possible de savoir s'il s'agit d'une transmission de textes ou d'un jeu littéraire.

Par cet ajout, la prophétie se charge d'un sombre et violent récit. La sibylle Tiburtine annonce aux sénateurs romains qu'un «serf volant et ligier » sera "guerroié » par un "goulpil renart plain de tricherie », qui l'évincera de «ses propres pastures ». Mais, poursuit-elle, les habitants d'une riche cité, considérant le dommage accompli par le renard, apporteront leur aide au cerf. Le «malicioux goupil» rassemblera alors des gens autour de lui pour commettre toutes sortes d'ignominies; en guise de réponse, le cerf, levant bravement les cornes, et accompagné des poussins de l'aigle, attaquera le renard pour lui couper la queue et les oreilles. Défait et honteux, le goupil ira se cacher. Mais un léopard averti de cette mutilation accourra pour détruire les bois et les pâtures du cerf. Levant à nouveau les cornes, ce dernier luttera contre le léopard "grox et enflé de sanc humain». À l'issue d'une grande bataille où les champs dégoutteront de sang, le félin, blessé, reprendra la route par la mer, mais une grande tempête le précipitera au fond de l'océan. D'autres «bestes sauvages » instruites de ces événements viendront délivrer le «lis qui estoit enclox par la cruauté du lepart» et iront dans la foulée détruire la « Grant Bretaigne ». La fin de la prophétie annonce le futur couronnement du cervidé, et renoue avec la fin du canevas latin de la prophétie : "cestuy serf prendra et recevra le sceptre d'or de la main du Tout Puyssant et sera couronné du dyademme royal».

\footnotetext{
${ }^{8}$ Différentes versions de cette prophétie sont consultables dans Christian JosTMANN, Sibilla Erithea Babilonica : Papsttum und Prophetie im 13. Jahrhundert, Hannover, Hahnsche Buchhandlung (Monumenta Germaniae Historica. Schriften, 54), 2006.

${ }^{9}$ Selon le découpage commode des éditeurs ; voir The Historia regum Britanniae of Geoffrey of Monmouth, I, Bern, Burgerbibliothek, MS 568, éd. Neil WrighT, Cambridge, D. S. Brewer, 1985 ; et, pour une traduction, Geoffroy de Monmouth. Histoire des rois de Bretagne, traduit et commenté par Laurence MATHEY-MAILLE, Paris, Belles Lettres, 1992 (La Roue à Livres).
} 
Apparaîtra alors, assez conventionnellement, 1'Antéchrist : "Adonc le Prince de iniquité vendra ou temple, Filz de perdicion, Chief d'orgueil, Mestre de malice, qui le monde mestra en subjection par les faux miracles qu'il fera. » La fin du monde sera arrivé. «De Sebile plus n'y a $\left(\mathrm{f}^{\circ} 97^{\mathrm{va}}\right)$.

Cette zoologie n'est pas mystérieuse pour un lecteur lettré et peut aisément être décryptée. L'auteur attend que l'on applique à ses phrases le même type d'exégèse que pour les vaticinations de Merlin. On s'en souvient, le petit prophète poilu avait lui-même donné la clé en révélant la signification des dragons rouge et blanc qui sommeillaient sous la tour de Vertigier - et d'illustres clercs avaient par la suite exercé leur sagacité sur le reste du texte, parfois avec une certaine méthode ${ }^{10}$.

L'affrontement d'un cerf issu de la «racine du lis » avec un léopard est une évocation indirecte de l'affrontement franco-anglais : le premier désigne Charles V (par transfert de l'emblème de prédilection de son fils Charles VI), et le second Édouard III. À ce symbolisme héraldique s'ajoute une expression prophétique, les «poucins de l'aigle » qui soutiennent les menées du cerf, qui renvoie à Bertrand Du Guesclin ${ }^{11}$. C'est de la part de la sibylle adopter un

\footnotetext{
${ }^{10}$ Je pense au commentaire d'Alain de Lille, présenté et analysé par Clara WILLE, «La symbolique animale de la Prophetia Merlini », Reinardus, 15 (2002), p. 175-190. De la même auteure, on peut lire "Le dossier des commentaires latins des Propheties Merlini », dans Moult obscures paroles, Études sur la Prophétie médiévale, recueillies par Richard TRACHSLER, avec la collaboration de Julien ABED et David EXPERT, Paris, Presses de l'université de Paris-Sorbonne, 2007, p. 167-184.

${ }^{11}$ Dans la partie finale du Songe de pestilence attribué à Henri de Ferrières (Les livres du roy Modus et de la royne Ratio, éd. Gunnar TILANDER, Paris, Société des anciens textes français, 1932, t. 2, p. 203-220), il est question d'une prophétie «trouvee en un livre que un tres grant clerc philosophe $i$ avoit mise comme vraie », selon laquelle un aigle aidé par des poussins agira en faveur de «Charles, le noble roi de France ». L'image de l'aigle parcourt également les poésies qu'Eustache Deschamps consacre au «tresbon Connestable » (par exemple le Lay du tresbon Connestable d'Eustache Deschamps, dans Euvres complètes, éd. Marquis de QueuX de SAINT-Hilaire et Gaston RaYNAUd, Paris, Firmin-Didot, 11 vol., 1878-1903 (SATF), ici t. II, p. 324-335).
} 
langage très proche du Songe de vergier ou des ballades politiques d'Eustache Deschamps, contemporains de la prophétie ${ }^{12}$.

Quant au renard, ce goupil malicieux et traître, dont l'origine est plus littéraire que prophétique, il semble figurer Charles II de Navarre, à l'instar du Renart de la Fiction du Lyon d'Eustache Deschamps (vers 1386) ${ }^{13}$. Dans ce poème allégorique inachevé sur la cour de Charles $\mathrm{V}$ (où le roi est figuré par Noble le Lion), Renart désigne Charles le Mauvais. Le poète champenois y dénonce ses agissements auprès d'Édouard III pour s'imposer face au roi de France; il évoque également la bataille de Cocherel (1365) qui mit fin à ses espoirs. Cette bataille décisive du conflit entre Charles II et le roi de France, où Bertrand Du Guesclin précipita le déclin militaire du Navarrais, pourrait bien être représentée par l'étrange mutilation de notre texte.

Les échos sont très frappants entre Eustache Deschamps et sibylle de notre manuscrit. Termes similaires pour brosser le portrait du personnage («soubtil, a tout barats et tricherie »), même provenance géographique du personnage :

Fiction du lyon: « Mais premiers parla a Renart, Qui venoit des marches d'Espagne » (v. 2642-2643)

ms Lyon 768 : « le serf legier en pluseurs lieux sera debouté et guerroié de ses prochains quar des parties d'Espaigne qui tiennent devers Gaule vendra un goupil renart plain de tricherie. » $\left(f^{\circ} 96^{v}\right)$

C'est la même violence qui gagne l'exposé des actions de cet animal maléfique. Deschamps le décrit semant sur sa route «Guerres, dissencions,

\footnotetext{
${ }^{12}$ Le refrain d'une ballade qui lui est attribuée exhorte ainsi à pleurer le bon connétable qui a su défendre «les fleurs de lis du lieupart felon ». Voir Eustache DeschamPS, op. cit., t. X, p. LXXIX.

${ }^{13}$ Eustache Deschamps, op. cit., t. VIII, p. 247-338. Sur ce texte, j'ai lu Maria NARBOnA CÁRCELES, «La contribution d'Eustache Deschamps à la construction du mythe de Charles, dit «le Mauvais », à partir de la Fiction du Lyon », dans Eustache Deschamps, témoin et modèle. Littérature et société politique (XIV $-X V I^{e}$ siècles), Paris, PUPS, 2008, p. 33-47.
} 
haines, / Traisons, contens, ataines, / Murmures et commocions / Forjurées rebellions » (v. 2705-2714). Frénésie de brutalité reprise dans la prophétie, où l'on suggère l'indicible de cette violence, lorsque le renard «fait aigres oultrages et faiz a ouïr orribles». La réécriture de la réalité (car la « prophétie politique »n'est rien d'autre qu'une forme de l'écriture de l'histoire) se double peu à peu d'une réinvention. La transposition animale permet même d'organiser dans le texte tout un réseau lexical de la sauvagerie qui se déploie dans la description des attitudes animales - signes de défi, marques d'agressivité - et des désordres climatiques.

L'horreur se poursuit par la vision du corps démembré, au moment où le lion secondé par les poussins de l'aigle «coupe[nt] au goupil la queue et les orailles ». Cette «tronsonliacion» rappelle fortement les prophéties de Merlin ${ }^{14}$. Selon un commentateur comme Alain de Lille, qui interprète avec systématisme les vaticinations, la droite désigne le spirituel, la gauche le temporel; un membre évoque plutôt un administrateur ou un conseiller; la queue est toujours interprétée comme l'armée qui suit un prince. La tradition d'exégèse, on le voit, a su produire de nouveaux textes plus de deux siècles après la composition de Geoffroy. Et les clés de lecture délivrées par l'activité herméneutique sont devenues des recettes d'écriture.

L'anthropomorphisation des animaux se signale par un goût pour les expressions abstraites, qui lève le voile sur la subjectivité du regard: la «tricherie du goupil», «l'orgueil et la felonnie du lepart» s'opposent à la noble attitude du cerf. Cette structure syntaxique (substantif abstrait suivi d'un complément de nom animal), récurrente, pourrait être une habitude des

\footnotetext{
${ }^{14}$ Aux paragraphes $42-44$, un rusé renard faisant le mort attire un imprudent sanglier et lui arrache le pied gauche, l'oreille droite et la queue.
} 
traducteurs de prophéties latines, manifestant la compréhension du texte qu'ils ont sous les yeux ${ }^{15}$.

Tout cela dépasse la simple peinture du rapport de force franco-anglais du $\mathrm{XIV}^{\mathrm{e}}$ siècle. Il est surtout question de la légitimité ou de l'illégitimité des actes de chacun. La prophétie dépasse la violence en la condamnant ou en la sublimant, et le langage codé, de fait, parle moins de sauvagerie et de brutalité que de justice et de paix. On retrouve là le rapport complexe du Moyen Âge à la violence : dénonciation de l'agressivité, mais en même temps justification de la vengeance pour le maintien de l'ordre ou de l'honneur, et recours ultime à la violence divine, qui met fin au désordre humain.

Le prisme animal, qui permet de renchérir sur l'effroi suscité par les massacres, permet ainsi de canaliser la violence : il l'ordonne en la présentant non comme une cruauté ponctuelle, mais comme un combat orienté dans l'histoire. Il donne sens au cours des événements, car l'ambition d'une telle prophétie n'est évidemment pas de contenir la violence en l'exprimant de manière imagée, mais bien de la diffuser, en créant des légendes noire (Charles de Navarre) et rose (Du Guesclin). À l'unisson de la propagande de la cour, la sibylle brosse le portrait d'un Charles qui restera jusqu'à nos jours «le Mauvais ». On pourrait s'étonner d'une telle instrumentalisation de la sibylle antique, mais le XIV ${ }^{\mathrm{e}}$ siècle offre bien d'autres exemples d'utilisation de la prophétie comme arme concrète et non comme vision religieuse ${ }^{16}$.

Le fait qu'un scribe ait intercalé une prophétie de nature politique si transparente (pour le milieu de la cour), dans le contenu relativement obscur de

\footnotetext{
${ }^{15}$ On consultera les abondantes remarques de Géraldine VEYSSEYRE, «"Metre en roman" les prophéties de Merlin. Voies et détours de l'interprétation dans trois traductions de l'Historia regum Britannie », dans Moult obscures paroles, op. cit., p. 107-166.

${ }^{16}$ Voir sur ce point Colette BEAUNE, «Perceforêt et Merlin. Prophétie, littérature et rumeurs au début de la guerre de Cent Ans », Fin du monde et signes des temps. Visionnaires et prophètes en France méridionale (fin XIII-début XIV ${ }^{e}$ siècle), Fanjeaux, Privat, 1992, (Cahiers de Fanjeaux 27).
} 
la parole de la sibylle Tiburtine, montre la concurrence de différents modes de lecture au sein d'un même texte. L'auteur attend de son lecteur qu'il sollicite l'histoire pour expliquer le texte, qu'il lise à travers des batailles animales la violence du monde humain. Autrement dit, qu'il rende le texte transparent et lui confère une valeur prophétique. Le scribe se pose sans doute moins en moralisateur privilégiant une approche spirituelle de l'histoire (ce qu'autorisent parfaitement les passages relatifs à la naissance du Christ ou à l'arrivée finale de l'Antéchrist), qu'en pur propagandiste.

Cette partie de la prophétie se retrouve en ouverture d'un manuscrit de Clermont-Ferrand (fin du XIV ${ }^{\mathrm{e}}$ siècle), mais la trame du songe (les neuf soleils) n'est pas reprise, malgré la rubrique identifiant ce texte comme une "prophétie de la sibylle Tiburtine $»^{17}$. Des prophéties voisines se trouvent dans un extrait d'un manuscrit de Tours (entre 1420 et 1440), où deux courts textes utilisant ce symbolisme sont placés dans la bouche de la sibylle, avant une version latine, conforme au modèle le plus répandu, de la Sibylla Tiburtina ${ }^{18}$.

Reste à imaginer comment ce texte était lu au cœur du manuscrit lyonnais contenant les deux premiers Pèlerinages de Guillaume de Diguleville. Les

${ }^{17}$ «Ce que s'enssuit est exstrait de prophecies de la dezime sibille, apellee en grejois Tiburtine, en latin Alburnee, autrement nommee Cassandra, fille qui fut au roy Priant de Troye " (Clermont-Ferrand, BM ms 249, $\mathrm{f}^{\circ} 3^{\mathrm{r}}$ ). Thierry LASSABATÈRE, "Diffusion et postérité de l'œuvre politique d'Eustache Deschamps, le témoignage des manuscrits », dans Thierry Lassabatère et Miren LACASSAGne, Les "Dictez vertueulx» d'Eustache Deschamps. Forme poétique et discours engagé à la fin du Moyen Âge, Paris, Presses de l'Université de Paris-Sorbonne, 2005, p. 107-120, évoque ce manuscrit p. 115-116.

${ }^{18}$ Il s'agit du ms Tours, BM 520. La rubrique générale annonçant ces trois textes est impayable : «La prophetie de Sebille la grant. La grant sebille prophetisa avant l'incarnacion iiii $B$.iii. ${ }^{c}$ ans, et a esté prise en .I. pillier de marbre a Romme, l'an de grace mil ccccii » $\left(\mathrm{f}^{\circ} 149^{\mathrm{v}}\right)$. Sur ce manuscrit, j'ai lu Matthew ToBIN, «Une collection de textes prophétiques du XV $\mathrm{XV}^{\mathrm{e}}$ siècle : le manuscrit 520 de la Bibliothèque de Tours ", dans Les Textes prophétiques et la prophétie en Occident (XII $-X V I^{e}$ siècle). Actes de la table ronde organisée par l'URA 1011 du CNRS et le Centre de Recherche "Histoire sociale et culturelle de l'Occident, XII -XVIII siècles " de l'Université de Paris X-Nanterre (Chantilly, 30-31 mai 1988), éd. André VAuchez, Palais Farnese, École Française de Rome, 1990, p. 127-133. L'auteur voit dans cette anthologie bilingue de textes prophétiques, qui a pour thème prédominant la Guerre de Cent ans, un «mélange mystérieux et fascinant » dépourvu de signification politique cohérente. 
versions françaises en prose (qui ne contiennent pas la partie consacrée aux animaux) se trouvent dans des contextes variés. Dans un manuscrit de Rennes, on la trouve dans une compilation de vulgarisation scientifique, comprenant l'Image du monde de Gossuin de Metz, le Livre du Trésor de Brunet Latin ${ }^{19}$. Dans un manuscrit de Paris, elle inaugure, avec une Apocalipsis latine, son commentaire français et le Livre de Seneke, un vaste ensemble de romans (Troie, Rou, Érec, Athis et Prophilias, Guillaume d'Engleterre, Ysle et Galeron, etc), qui se clôt sur Neuf miracles de Notre Dame ${ }^{20}$. Cet ensemble (ApocalypseTiburtine-Sénèque) se retrouve dans un chansonnier aujourd'hui conservé en deux morceaux à Londres et à Oxford ${ }^{21}$. Signalons enfin deux manuscrits disparus qui figuraient dans la bibliothèque de Charles $\mathrm{V}$ (dont témoignent deux catalogues d'avant 1411) : l'un faisait se côtoyer les «Moralitez du Jeu des eschez, les propheciez Merlin et les propheciez Sebille, la Table Salmon, les propheciez Methodez», le second «Du Saint Graal, de la creacion Adam, la Naissance de toutes choses, Merlin, les Prophecies Sebile, les Prophecies Methode, evesque de Patras, en prose, escript de lettre de forme, en françois $»^{22}$. Dans ces environnements divers, on peut imaginer que le texte constituait tantôt une lecture dévote, tantôt un document sur la divination, conservé par curiosité,

\footnotetext{
${ }^{19}$ Rennes, Bibliothèque municipale 593, $\mathrm{f}^{\circ}$ 163-165 (début du XIV ${ }^{\mathrm{e}}$ siècle) ; édité dans $L a$ Prophétie de la Sibylle Tiburtine, éd. Jeanne BAROIN et Josiane HAFFEn, op. cit., p. 113-135. Pour les jolies images du manuscrit, voir Sophie CASSAGNES-BROUQUET, L'Image du monde. Un trésor enluminé de la bibliothèque de Rennes, Rennes, Presses Universitaires de Rennes / Bibliothèque de Rennes Métropole, 2003.

${ }^{20}$ Paris, BnF fr. 375, fo 27-28 (copié en 1288) ; édité dans La Prophétie de la Sibylle Tiburtine, éd. Jeanne BAROIN et Josiane HAFFEN, op. cit., p. 85-111.

${ }^{21}$ Londres, Brit. Libr., Harley 4972 et Oxford, Bodl. Douce 308. Copié à Metz au début du $\mathrm{XIV}^{\mathrm{e}}$ siècle, il contient le Tournoi de Chauvency, les Voeux du paon, un vaste chansonnier de 512 pièces, le Bestiaire d'amour de Richard de Fournival et la Response (vers 1285), et enfin le Tournoiement Antichrist de Huon de Méry. Je remercie Mme Nancy F. Regalado de m'avoir fait parvenir les photocopies du microfilm, qui m'ont permis de vérifier l'indication exacte de J. Haffen et J. Baroin selon laquelle le texte a été copié sur l'exemplaire de Paris.

${ }^{22}$ Voir Léopold DeLISLE, Recherches sur la librairie de Charles V, roi de France, 1337-1380. Partie II : inventaire général des livres ayant appartenu aux rois Charles V et Charles VI et à Jean, duc de Berry, notes et tables, Amsterdam, Gérard Th. Van Heusden, 1967 (1 ${ }^{\text {ère }}$ éd. Paris, 1907), respectivement p. 91 et 183.
} 
plus rarement un texte historique. Dans ces perspectives, la prophétie, avec sa longue liste d'initiales, restait majoritairement obscure, hormis la brève partie consacrée à la naissance du Christ.

Dans le manuscrit de Lyon, en revanche, le texte n'est pas par essence hermétique. Le remplacement d'une partie a priori obscure par des allusions à la Guerre de Cent ans révèle le désir de rendre accessible la quasi-totalité du discours sibyllin. Cette problématique (lecture / non-lecture) est formulée par les études littéraires actuelles sur la prophétie ${ }^{23}$. Elle permet d'expliquer que des lecteurs médiévaux, selon le manuscrit qu'ils avaient sous les yeux, aient ressenti la même parole de la sibylle tantôt comme un langage essentiellement obscur, tantôt comme une parole essentiellement transparente ${ }^{24}$.

Qu'en est-il dans notre manuscrit de Lyon? La Tiburtine est le premier des deux textes prophétiques insérés, peut-être sur un cahier autonome ${ }^{25}$, de la première version du Pèlerinage de Vie humaine, plus précisément entre les vers 13482 et 13483 de l'édition Stürzinger ${ }^{26}$. Guillaume de Diguleville, luimême auteur d'un Roman de la fleur de lis (vers 1338), vision en forme d'allégorie politique exaltant la royauté française et démontrant sa primauté sur

\footnotetext{
${ }^{23}$ Elle constitue l'une des problématiques centrales de la thèse de Anke Holdenried.

${ }^{24}$ «Leurs parolles sont aucunes fois doubles, amphiboliques, a .II. visages », écrit Nicole Oresme dans son Livre de Divinacions. Il «sembloit que ce fussent si comme croniques de choses passees et ja avenues, tant estoient clers et entendibles et plains leurs dis et escrips ", dira Droiture à Christine de Pizan. Voir Nicole ORESME, Livre de Divinacions, éd. Sylvie LEFÈVRE, mémoire de DEA, cité par Jacqueline CERQuiglini-Toulet, «Polysémie, ambiguiité et équivoque dans la théorie et la pratique poétiques du Moyen Âge français », dans L'Ambiguïté. Cinq études historiques, éd. Irène RosiER, Lille, Presses universitaires de Lille, 1988, ici p. 171 ; et Christine DE PIZAN, La Città delle dame, éd. Earl Jeffrey RichaRds, trad. en italien de Patricia CARAFFI, Milan, Luni, 1997, p. 220.

${ }^{25}$ Je n'ai consulté que le microfilm conservé à l'IRHT. Ce cahier aurait-il pu provenir de l'un des deux manuscrits perdus de la bibliothèque de Charles $\mathrm{V}$ ?

${ }^{26}$ Guillaume De Digulleville, Le Pèlerinage de vie humaine, éd. Johann Jakob STÜRZINGER, Londres, Nichols and Sons, Roxburghe Club, 1893, p. 420-421. L'éditeur a signalé en note de bas de page l'existence de ces deux textes.
} 
le léopard (anglais) et l'aigle à double tête (germanique) ${ }^{27}$, ne semble pas à l'origine de ces textes. Peut-être faut-il revenir au récit même du Pèlerinage de Vie humaine. À la fin de l'œuvre, le pèlerin a rencontré deux femmes allégoriques, Vieillesse et Infirmité, puis voit la Mort s'approcher de son lit. Dans un ultime discours, Grâce de Dieu convainc le pèlerin qu'il lui faut quitter son corps pour effectuer le voyage allégorique qui occupera l'œuvre suivante. La proximité de l'image de la vieillesse, du thème du passage dans l'autre monde et de la conception eschatologique peut nous évoquer la sibylle de Cumes, cette autre vieille qui révèle à Énée les secrets de la route vers les morts et promet un savoir sur l'au-delà. Il serait périlleux de vouloir montrer que l'insertion d'un cahier au sein d'un manuscrit du Pèlerinage de Vie humaine ${ }^{28}$ est un écho de cette ancienne place dévolue à la sibylle. À ma connaissance, même s'il est tentant de faire ce rapprochement, aucun voyage allégorique vers l'au-delà n'instaure une figure de sibylle virgilienne à l'entrée de l'autre monde ${ }^{29}$.

Un passage de l'Ovide moralisé instille pourtant un doute. Le récit de la rencontre entre Énée et la sibylle de Cumes est en effet suivi chez Ovide de la confession de la prophétesse qui expose par un beau mythe les raisons de son extrême vieillesse ${ }^{30}$. Mais l'auteur médiéval ne se contente pas de la

\footnotetext{
${ }^{27}$ Arthur PIAGET, «Un roman inédit de Guillaume de Diguleville : Le Roman de la Fleur de lis », Romania, 62 (1936), p. 317-358 (numérisé en mode texte sans l'apparat critique sur le CD-Rom Corpus de littérature médiévale, Ferney-Voltaire, Champion, 2001).

${ }^{28}$ Géraldine VEYSSEYRE avance le chiffre de 77 copies dans « Lecture linéaire ou consultation ponctuelle ? Structuration du texte et apparats dans les manuscrits des Pèlerinages ", dans Guillaume de Diguleville. Les Pèlerinages allégoriques, sous la direction de Frédéric DuVAL et Fabienne PoMEL, Rennes, Pressses universitaires de Rennes (coll. Interférences), 2008, p. 315-330.

${ }^{29}$ Sur cette vaste littérature, j'ai consulté Fabienne POMEL, Les voies de l'au-delà et l'essor de l'allégorie au Moyen Âge, Paris, Champion (Nouvelle Bibliothèque du Moyen Âge, 57), 2001. Voir aussi Mattia Cavagna, "Enfer et purgatoire dans le Pèlerinage de l'âme de Guillaume de Diguleville, entre tradition et innovation », dans Guillaume de Diguleville, op. cit., p. 111-130.

${ }^{30}$ Sur ce beau mythe, voir Jacqueline CERQUIGLINI-TOULET, «Écho et sibylle, la voix féminine au Moyen Âge : entre affirmation et extinction », Équinoxe : revue romande de
} 
moralisation de cette fable; il l'augmente d'un court rappel sur l'histoire des sibylles, d'une version octosyllabique de la prophétie de la sibylle Tiburtine, et d'un poème sur les Signes du Jugement. Agencement de matières païennes et chrétiennes, qui illustre bien en ce milieu de $\mathrm{XIV}^{\mathrm{e}}$ siècle l'ambiguité foncière de toute représentation de la sibylle, personnage mythologique mais prophétique, appartenant mais échappant à la fabula.

L'apparition de petites vieilles ${ }^{31}$ à l'orée d'un passage dans l'autre monde suffisait-elle, par une association d'idées analogue à celle de l'Ovide moralisé, à ressusciter l'image de la sibylle de Cumes, et à indiquer un emplacement possible pour une prophétie politique?

Déchiffrer des batailles d'animaux, lire un récit imagé de la violence contemporaine, et ce, au milieu même d'une œuvre édifiante, témoigne du goût des lecteurs médiévaux pour l'intrusion de textes peignant la réalité au sein même d'œuvres qui entendent s'en échapper. Ces migrations textuelles, qui font entendre par la voix des plus anciens prophètes le récit de ce qui se passe à la porte, ne sont pas le moindre chemin d'accès au monde fascinant des manuscrits médiévaux.

sciences humaines, 23, Le Genre de la voix (automne 2002), Genève, p. 81-91. Sur l'épisode où intervient la sibylle, on peut lire Karl SNEYDERS DE VOGEL, "Ovide moralisé, XIV, 10671716 », Neophilologus, 28, 1 (décembre 1943), p. 88-106, et Anna Maria BABBI, « La sibylle dans l'Ovide moralisé ", dans Catherine Bel, Pascale Dumont et Frank WiLlaert (éds), «Contez me tout ». Mélanges de langue et de littérature médiévales offerts à Herman Braet, Louvain, Peeters, 2006 (coll. La République des Lettres, 28), p. 423-434.

${ }^{31}$ Voir sur ce point l'article d'Anne-Laure LALlouetTE, « La vieillesse dans le Pèlerinage de vie humaine », dans Guillaume de Diguleville, op. cit., p. 215-228. 